\title{
Chemical composition, antioxidant and anticholinesterase potentials of essential oil of Rumex hastatus D. Don collected from the North West of Pakistan
}

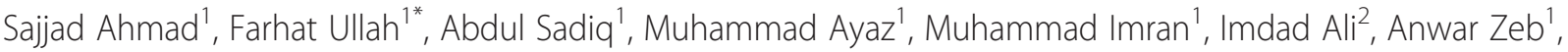
Farman Ullah ${ }^{3}$ and Muhammad Raza Shah ${ }^{2}$

\begin{abstract}
Background: Ethnomedicinally Rumex hastatus D. Don has been used since long for various ailments especially in neurological disorders. The reported data and the importance of Rumex genus demonstrate the vital medicinal value of $R$. hastatus.

Methods: In the current investigational study, isolation of essential oil and its antioxidant and anticholinesterase assays were performed. The essential oil of R. hastatus was analyzed by GC-MS for the first time. The essential oil was evaluated for anticholinesterase and antioxidant assays. The anticholinesterase assay was conducted at various concentrations (62.5 to $1000 \mathrm{\mu g} / \mathrm{ml}$ ) against acetylcholinesterase (AChE) and butyrylcholinesterase (BChE). Similarly, the antioxidant potential was determined using DPPH and ABTS free radicals.

Results: The GC-MS analysis of essential oil showed 123 components. The result recorded for the anticholinesterase assays demonstrated a marked potential against $A C h E$ and $B C h E$ with $I C_{50}$ values of 32.54 and $97.38 \mu \mathrm{g} / \mathrm{ml}$ respectively which were comparable with the positive control i.e., galanthamine (AChE, $I C_{50}=4.73 \mu \mathrm{g} / \mathrm{ml}$ and $B C h E, I C_{50}=11.09 \mu \mathrm{m} /$ $\mathrm{ml}$ ). The antioxidant assays against DPPH and ABTS free radicals also exhibited significant scavenging potential with $I C_{50}$ values of 3.71 and $6.29 \mu \mathrm{g} / \mathrm{ml}$ respectively, while for ascorbic acid the $I C_{50}$ value was $<0.1 \mu \mathrm{g} / \mathrm{ml}$ against both free radicals.

Conclusions: Based on the current investigational studies, it may be concluded that $R$. hastatus is an effective source of essential oil's components having anticholinesterase and antioxidant potentials, which after subjecting to drug development may lead to novel drug candidates against neurodegenerative disorders.
\end{abstract}

Keywords: Essential oil, Acetylcholinesterase, Butyrylcholinesterase, Antioxidant, GC-MS, Free radicals, Rumex hastatus

\section{Background}

A brief history of medicine demonstrates the use of herbal medicine for the effective treatment of various ailments. Herbal medicine has been used since long in various forms including the decoction, powdered sample, oleoresins, crude extracts, fixed oil, essential oil etc [1]. Various plants have been used in multiple types of food

\footnotetext{
* Correspondence: farhataziz80@hotmail.com

'Department of Pharmacy, University of Malakand, Chakdara 18000, Dir (L), KPK, Pakistan

Full list of author information is available at the end of the article
}

items for preservation and therapeutic effects [2]. In this regards, essential oils have been manifested by several reporters to play a major role. Essential oils have the property to attenuate the effects of free radicals, e.g, reactive oxygen species (ROS) which are derived from metabolism of oxygen and exogenous agents [3]. ROS are responsible for wide variety of diseased conditions including oxidative stress and nervous disorders [4]. Essential oils are well-known for their radicals scavenging properties and amelioration of various cognitive disorders. Among the cognitive disorders, Alzheimer's disease 
(AD) is the most common in elderly people [5]. One of the best therapeutic approaches for $\mathrm{AD}$ is to increase the concentration of the neurotransmitter (Acetylcholine) by inhibiting the enzyme (acetylcholinesterase) responsible for its breakdown. Various drugs originated either from natural or synthetic sources are being used for the management of $\mathrm{AD}$ and other nervous disorders [6]. Similarly, it has also been reported that oxidative stress are responsible for wide variety of mental diseases due to neuronal degeneration and other factors. Oxidative stress is mainly developed due to increase in concentration of free radicals within the body. The free radicals have been reported by numerous researchers to possess multiple destructive properties, due to which interest has been focused to scavenge the free radicals somehow and avoid their deteriorating effects [7]. In this context, investigators are trying to explore more and more sources of natural and synthetic bioactive principles [8]. The natural drugs are being preferred over the synthetic due to their negligible harmful and deleterious effects [9]. That's why researchers are trying to explore novel sources of natural medicine [10-18]. Among the natural sources, herbal medicines have been shown promising results due to the presence of numerous secondary metabolites and essential oils. Essential oils isolated from various plants have been reported to possess marked acetylcholinesterase inhibitory and radicals scavenging potential [19-21]. Traditional knowledge also demonstrates the use of essential oils for various nervous system disorders [22].

$R$. hastatus D. Don belongs to the family Polygonaceae. Various members of this family have been reported to be used against paralysis, headache and other nervous system disorders [23-26]. Various solvent samples of $R$. hastatus have recently been reported to possess strong anticholinesterase and antioxidant potentials [26]. To date, the chemical composition of essential oil of $R$. hastatus has not been reported or evaluated for any pharmacological activity. Based on the literature survey and medicinal importance of $R$. hastatus, the current investigational study is arranged to isolate the essential oil, analyze the chemical composition and to evaluate for the anticholinesterase and antioxidant potentials, which may be a possible remedy for oxidative stress and nervous system disorder.

\section{Methods}

\section{Plant sample collection}

The aerial parts of $R$. hastatus were collected from the proximity of University of Malakand. The plant was identified by plant taxonomist Ali Hazrat and deposited with voucher number (1015SJ) in the herbarium of Department of Botany, Shaheed Benazir Bhutto University Sheringal, Dir (U), KPK, Pakistan. Extraction of essential oil of $R$. hastatus was performed by hydrodistillation using clevenger type apparatus [27]. The essential oil obtained was stored at $-20{ }^{\circ} \mathrm{C}$ until required.

\section{Chemicals and drugs}

DPPH (Sigma Aldrich CHEMIE GmbH USA, code 101341986), $\mathrm{K}_{2} \mathrm{~S}_{2} \mathrm{O}_{4}$ (Riedel-de Haen Germany), ABTS (Sigma Aldrich USA, code 1001551916), Gallic acid (GmbH USA), Folin Ciocalteu reagent (Merck Co. Germany). AChE (Electric eel type-VI-S, Sigma-Aldrich GmbH USA, code 1001596210), BChE (Equine serum Lyophilized Sigma-Aldrich GmbH USA, code 101292670), Acetylthiocholine iodide (Sigma-Aldrich UK, code 101303874), Butyrylthiocholine Iodide (Sigma-Aldrich Switzerland, code 101334643), DTNB (Sigma-Aldrich Germany, code 101261619), Galanthamine hydrobromide Lycoris Sp. (Sigma-Aldrich France, code G1660). $\mathrm{K}_{2} \mathrm{HPO}_{4}, \mathrm{KH}_{2} \mathrm{PO}_{4}, \mathrm{KOH}$. All the chemical used were of analytical grade.

\section{Gas Chromatography (GC) analysis}

The GC analysis of essential oil was carried out via gas chromatograph Agilent USB-393752 (Agilent Technologies, Palo Alto, CA, USA) with HHP-5MS $5 \%$ phenylmethyl siloxane capillary column $(30 \mathrm{~m} \times 0.25 \mathrm{~mm} \times$ $0.25 \mu \mathrm{m}$ film thickness; Restek, Bellefonte, PA) connected with FID detector. The oven was set at temperature of $70{ }^{\circ} \mathrm{C}$ for one minute and then increased to $180{ }^{\circ} \mathrm{C}$ at the rate of $6{ }^{\circ} \mathrm{C} / \mathrm{min}$ for $5 \mathrm{~min}$ and lastly to $280{ }^{\circ} \mathrm{C}$ at the rate of $5{ }^{\circ} \mathrm{C} / \mathrm{min}$ for $20 \mathrm{~min}$. The temperature of injector and detector were maintained at $220^{\circ} \mathrm{C}$ and $290^{\circ} \mathrm{C}$ correspondingly. The flow rate of carrier gas i.e., Helium was $1 \mathrm{ml} / \mathrm{min}$ and the diluted samples $(1 / 1000$ in $n$-pentane, v/v) of $1 \mu$ l were manually injected in the split-less mode.

\section{Gas Chromatography-Mass Spectrometry (GC-MS) analysis}

The GC/MS of the essential oil was performed via USB-393752 gas chromatograph (Agilent Technologies, Palo Alto, CA, USA) with a HHP-5MS $5 \%$ phenylmethyl siloxane capillary column $(30 \mathrm{~m} \times 0.25 \mathrm{~mm} \times$ $0.25 \mu \mathrm{m}$ film thickness; Restek, Bellefonte, PA) outfitted with an Agilent HP-5973 mass selective detector in the electron impact mode (Ionization energy: $70 \mathrm{eV}$ ) working under the experimental conditions as those maintained for GC.

\section{Identification of components}

The recognition of all the major constituents of oil was performed by comparing their retention times with the authentic compounds in the literature. Identification of compounds was further processed through the spectral data obtained from the Wiley and NIST libraries as well 
Table 1 Anticholinesterase activity of essential oil of Rumex hastatus at various concentrations

\begin{tabular}{|c|c|c|c|c|c|c|c|}
\hline Samples & Enzymes & $\begin{array}{l}\text { Conc. } \mu \mathrm{g} / \mathrm{ml} \\
62.5\end{array}$ & $\begin{array}{l}\text { Conc. } \mu \mathrm{g} / \mathrm{ml} \\
125\end{array}$ & $\begin{array}{l}\text { Conc. } \mu \mathrm{g} / \mathrm{ml} \\
250\end{array}$ & $\begin{array}{l}\text { Conc. } \mu \mathrm{g} / \mathrm{ml} \\
500\end{array}$ & $\begin{array}{l}\text { Conc. } \mu \mathrm{g} / \mathrm{ml} \\
1000\end{array}$ & $\begin{array}{l}\mathrm{IC}_{50} \mathrm{\mu g} / \\
\mathrm{ml}\end{array}$ \\
\hline$\overline{\mathrm{EO}}$ & AChE & $54.32 \pm 1.33$ & $61.64 \pm 1.60$ & $67.26 \pm 1.24$ & $71.70 \pm 1.63$ & $74.90 \pm 0.52$ & 32.54 \\
\hline EO & BChE & $46.32 \pm 3.50$ & $52.73 \pm 0.78$ & $57.00 \pm 2.80$ & $66.33 \pm 0.49$ & $71.32 \pm 4.8$ & 97.38 \\
\hline Gal & AChE & $72.08 \pm 1.04$ & $78.58 \pm 1.12$ & $83.70 \pm 1.60$ & $89.00 \pm 1.15$ & $96.65 \pm 1.34$ & 04.73 \\
\hline Gal & BChE & $66.87 \pm 1.27$ & $73.67 \pm 0.88$ & $79.95 \pm 2.01$ & $86.62 \pm 1.67$ & $91.61 \pm 0.43$ & 11.09 \\
\hline
\end{tabular}

Data is expressed as Mean \pm SEM; EO and Gal are abbreviated for Essential oil and Galanthamine respectively

as fragmentation patterns' comparisons of the mass spectra with data reported in literature or with those of mass spectra from literature $[28,29]$. Each determination was processed in duplicate.

\section{Anticholinesterase assays}

Anticholinesterase (AChE and BChE inhibitions) activity was performed for the essential oil of $R$. hastatus by spectrophotometric analysis following the method of Ellman's assay [30]. The substrates used were acetylthiocholine iodide and butyrylthiocholine iodide. Briefly, $5 \mu \mathrm{L}$ of $0.03 \mathrm{U} / \mathrm{mL}$ AChE and $0.01 \mathrm{U} / \mathrm{mL}$ BChE were taken in a cuvette and $205 \mu \mathrm{L}$ of essential oil having concentration of $62.5-1000 \mu \mathrm{g} / \mathrm{mL}$ were transferred to them using micropipette. Similarly, $5 \mu$ Lof DTNB was also added to this afterwards. The mixtures obtained were kept in water bath for $15 \mathrm{~min}$ at the temperature of $30{ }^{\circ} \mathrm{C}$. After incubation, $5 \mu \mathrm{L}$ of the Substrates were added to the mixture to optimize the reaction. A double beam spectrophotometer was used to measure the reaction time at $412 \mathrm{~nm}$ via a double beam spectrophotometer (Thermo electron corporation USA). Absorption values were obtained for $4 \mathrm{~min}$. Meanwhile, the yellow colored mixtures indicated the formation of 5-thio-2-nitrobenzoate anion as a reaction product of thiocholines and DTNB. White assay was also performed without enzymes and plant samples to check the non-enzymatic hydrolysis of substrate. The mixture which contained all the components excluding essential oil was marked as control. Percent enzyme activity and percent inhibition were recorded as follows.

$$
\begin{aligned}
& \mathrm{V}=\frac{\Delta \mathrm{Abs}}{\Delta \mathrm{t}} \\
& \% \text { enzyme activity }=\frac{\mathrm{V}}{\mathrm{V}_{\max }} \times 100 \\
& \% \text { enzyme inhibition }=100-\% \text { enzyme activity }
\end{aligned}
$$

(Where V symbolizes the rate of reaction in the presence of inhibitor and $V_{\max }$ stands for rate of reaction without inhibitor)

\section{DPPH radical scavenging assay}

The DPPH radical scavenging potential was evaluated for essential oil of $R$. hastatus following previously described procedure [31]. DPPH solution (0.004 \%) was prepared in methanol to get a deep violet colored solution. Similarly, stock solution of essential oil was prepared in ethanol having concentration of $1 \mathrm{mg} /$ $\mathrm{mL}$. The stock solution was serially diluted to get the

\begin{tabular}{|c|c|c|c|c|c|c|c|}
\hline $\mathrm{RT}$ (min) & Height & Height (\%) & Area & Area (\%) & Area Sum \% & Base Peak m/z & Width \\
\hline 6.447 & 254413 & 18.51 & 620057 & 20.82 & 5.87 & 83 & 0.127 \\
\hline 6.818 & 324110 & 23.59 & 626045 & 21.02 & 5.93 & 57.1 & 0.077 \\
\hline 10.958 & 430958 & 31.36 & 822529 & 27.61 & 7.79 & 55.1 & 0.074 \\
\hline 11.363 & 250143 & 18.2 & 592697 & 19.9 & 5.61 & 59.1 & 0.09 \\
\hline 11.761 & 278058 & 20.23 & 665761 & 22.35 & 6.31 & 59.1 & 0.094 \\
\hline 12.97 & 177060 & 12.88 & 399792 & 13.42 & 3.79 & 43.1 & 0.097 \\
\hline 13.171 & 312841 & 22.77 & 664487 & 22.31 & 6.29 & 55.1 & 0.08 \\
\hline 13.308 & $1 E+06$ & 100 & $3 E+06$ & 100 & 28.21 & 57.1 & 0.1 \\
\hline 15.063 & 159790 & 11.63 & 336861 & 11.31 & 3.19 & 55.1 & 0.08 \\
\hline 19.213 & 450356 & 32.77 & 782083 & 26.26 & 7.41 & 133.1 & 0.064 \\
\hline
\end{tabular}
concentrations of 62.5 to $1000 \mu \mathrm{g} / \mathrm{mL}$. Afterwards,

Table 2 Parameters of various components of essential oil of Rumex hastatus 
Table 3 List of components of essential oil of Rumexhastatus

\begin{tabular}{|c|c|c|c|c|c|}
\hline S.No & Compound Label & Common name & RT & Formula & Hits (DB) \\
\hline 1. & Trans-dideuterioxy-cyclopentene & NF & 5.757 & $\mathrm{C} 5 \mathrm{H} 6 \mathrm{D} 2 \mathrm{O} 2$ & 10 \\
\hline 2. & 1-Nonen-4-ol & NF & 5.884 & $\mathrm{C} 9 \mathrm{H} 18 \mathrm{O}$ & 10 \\
\hline 3. & Ethyl 2-hydroxybutyrate & NF & 6.169 & $\mathrm{C} 6 \mathrm{H} 12 \mathrm{O} 3$ & 10 \\
\hline 4. & 2(5H)-Furanone, 5-ethyl & NF & 6.445 & $\mathrm{C} 6 \mathrm{H} 8 \mathrm{O} 2$ & 10 \\
\hline 5. & Pentanoic acid, 4-oxo & Levulinic acid & 6.68 & $\mathrm{C} 5 \mathrm{H} 8 \mathrm{O} 3$ & 10 \\
\hline 6. & 2,2-Dimethylpropanoic anhydride & Trimethylacetic anhydride & 6.819 & $\mathrm{C} 10 \mathrm{H} 18 \mathrm{O} 3$ & 10 \\
\hline 7. & Heptanoic acid & Enanthic acid & 7.117 & $\mathrm{C} 7 \mathrm{H} 14 \mathrm{O} 2$ & 10 \\
\hline 8. & Ethanethioic acid, S-(2-methylpropyl) ester & NF & 7.374 & $\mathrm{C} 6 \mathrm{H} 12 \mathrm{OS}$ & 10 \\
\hline 9. & 4-Octanol, 7-methyl & NF & 7.511 & $\mathrm{C} 9 \mathrm{H} 20 \mathrm{O}$ & 10 \\
\hline 10. & 4-(Tetrahydrofuranyl-2-oxy)-4-methyl-2-pentanone & NF & 7.619 & $\mathrm{C} 10 \mathrm{H} 18 \mathrm{O} 3$ & 10 \\
\hline 11. & Cyclopropane, 1,2-dimethyl-1-pentyl & NF & 7.698 & $\mathrm{C} 10 \mathrm{H} 20$ & 10 \\
\hline 12. & n-Nonanal & Nonanal & 7.852 & $\mathrm{C} 9 \mathrm{H} 18 \mathrm{O}$ & 10 \\
\hline 13. & Cyclooctanone & NF & 8.275 & $\mathrm{C} 8 \mathrm{H} 14 \mathrm{O}$ & 10 \\
\hline 14. & 1,4,4-Trimethylcyclohexa-2-en-1-ol & NF & 8.494 & $\mathrm{C} 9 \mathrm{H} 16 \mathrm{O}$ & 10 \\
\hline 15. & 3-Octanol, 2-methyl & NF & 8.716 & $\mathrm{C} 9 \mathrm{H} 20 \mathrm{O}$ & 10 \\
\hline 16. & 2-Oxatricyclo[3.3.1.1(3,7)]decane, 1-methyl- & NF & 9.116 & $\mathrm{C} 10 \mathrm{H} 16 \mathrm{O}$ & 10 \\
\hline 17. & Succinimide, N-methoxy & NF & 9.338 & $\mathrm{C} 5 \mathrm{H} 7 \mathrm{NO} 3$ & 10 \\
\hline 18. & 4-Heptanol, 2-methyl & NF & 9.547 & $\mathrm{C} 8 \mathrm{H} 18 \mathrm{O}$ & 10 \\
\hline 19. & Ethanone, 1-(methylphenyl) & Methylacetophenone & 9.712 & $\mathrm{C} 9 \mathrm{H} 100$ & 10 \\
\hline 20. & Decanal & NF & 10.099 & $\mathrm{C} 10 \mathrm{H} 200$ & 10 \\
\hline 21. & 3-Heptanol, 2,4-dimethyl & NF & 10.328 & $\mathrm{C} 9 \mathrm{H} 20 \mathrm{O}$ & 10 \\
\hline 22. & Cyclooctanone & NF & 10.957 & $\mathrm{C} 8 \mathrm{H} 14 \mathrm{O}$ & 10 \\
\hline 23. & 1-Decyne (CAS) \$\$ Octylacetylene & NF & 11.165 & $\mathrm{C} 10 \mathrm{H} 18$ & 10 \\
\hline 24. & 3-Heptanol, 5-methyl & NF & 11.364 & $\mathrm{C} 8 \mathrm{H} 18 \mathrm{O}$ & 10 \\
\hline 25. & Nonanoic acid & Pelargic acid & 11.456 & $\mathrm{C} 9 \mathrm{H} 18 \mathrm{O} 2$ & 10 \\
\hline 26. & ETHYL AMYL CARBINOL & NF & 11.763 & $\mathrm{C} 8 \mathrm{H} 18 \mathrm{O}$ & 10 \\
\hline 27. & CIS-SABINENE HYDRATE & NF & 11.96 & $\mathrm{C} 10 \mathrm{H} 18 \mathrm{O}$ & 10 \\
\hline 28. & 1,8-Bisoxiranylnonane & NF & 12.047 & $\mathrm{C} 13 \mathrm{H} 24 \mathrm{O} 2$ & 10 \\
\hline 29. & 3-Heptanone, 4-methyl & NF & 12.817 & $\mathrm{C} 8 \mathrm{H} 16 \mathrm{O}$ & 10 \\
\hline 30. & Methyl 2-vinylbutanoate & NF & 12.972 & $\mathrm{C} 7 \mathrm{H} 12 \mathrm{O} 2$ & 10 \\
\hline 31. & trans-3-Nonen-2-one & NF & 13.171 & $\mathrm{C} 9 \mathrm{H} 16 \mathrm{O}$ & 10 \\
\hline 32. & Octane, 2,4,6-trimethyl & NF & 13.309 & $\mathrm{C} 11 \mathrm{H} 24$ & 10 \\
\hline 33. & 2H-Pyran-2-one, 6-heptyltetrahydro & Delta.-laurolactone & 13.471 & $\mathrm{C} 12 \mathrm{H} 22 \mathrm{O} 2$ & 10 \\
\hline 34. & Decanoic acid & Capric acid & 13.601 & $\mathrm{C} 10 \mathrm{H} 20 \mathrm{O} 2$ & 10 \\
\hline 35. & 3-Octanol & NF & 14.002 & $\mathrm{C} 10 \mathrm{H} 22 \mathrm{O}$ & 10 \\
\hline 36. & Ethyl 3,3-dimethylbutyrate & NF & 14.246 & $\mathrm{C} 8 \mathrm{H} 16 \mathrm{O} 2$ & 1 \\
\hline 37. & 5-Hexenal & NF & 14.547 & $\mathrm{C} 6 \mathrm{H} 100$ & 10 \\
\hline 38. & 2-Pentenoic acid, 4-hydroxy & NF & 14.878 & $\mathrm{C} 5 \mathrm{H} 8 \mathrm{O} 3$ & 10 \\
\hline 39. & Nonanoic acid, 9-oxo-, methyl ester & Azelaadehydic acid & 15.065 & $\mathrm{C} 10 \mathrm{H} 18 \mathrm{O} 3$ & 10 \\
\hline 40. & Thiophene, 2-methoxy & $\mathrm{NF}$ & 15.345 & $\mathrm{C} 5 \mathrm{H} 6 \mathrm{OS}$ & 3 \\
\hline 41. & Octanoic acid, 8-hydroxy & NF & 15.49 & $\mathrm{C} 8 \mathrm{H} 16 \mathrm{O} 3$ & 10 \\
\hline 42. & Oxirane, octyl & NF & 15.604 & $\mathrm{C} 10 \mathrm{H} 20 \mathrm{O}$ & 10 \\
\hline 43. & Butane, 1,1'-oxybis[3-methyl & NF & 15.875 & $\mathrm{C} 10 \mathrm{H} 22 \mathrm{O}$ & 5 \\
\hline 44. & 3-Hydroxy-4-methoxystyrene & NF & 16.153 & $\mathrm{C} 9 \mathrm{H} 10 \mathrm{O} 2$ & 7 \\
\hline
\end{tabular}


Table 3 List of components of essential oil of Rumexhastatus (Continued)

\begin{tabular}{|c|c|c|c|c|c|}
\hline 45. & Octanoic Acid & n-Caprylic acid & 16.355 & $\mathrm{C} 8 \mathrm{H} 16 \mathrm{O} 2$ & 10 \\
\hline 46. & 3-Hexanol, 3,5-dimethyl & NF & 16.55 & $\mathrm{C} 8 \mathrm{H} 18 \mathrm{O}$ & 10 \\
\hline 47. & 2-Tridecen-1-ol, (E) & NF & 16.643 & $\mathrm{C} 13 \mathrm{H} 26 \mathrm{O}$ & 10 \\
\hline 48. & 1-Isopropyl-4,7-dimethyl-1,2-dihydronaphthalene & Alpha-Calcorene & 16.877 & $\mathrm{C} 15 \mathrm{H} 20$ & 10 \\
\hline 49. & 4-(5',5'-dimethyl-2'-methylidene-3',8'-dioxabicyclo[5.1.0]oct-4-ylidene)-2-b... & NF & 17.084 & $\mathrm{C} 13 \mathrm{H} 18 \mathrm{O} 3$ & 5 \\
\hline 50. & 9-Methyl-S-octahydrophenanathracene & NF & 17.192 & $\mathrm{C} 15 \mathrm{H} 20$ & 10 \\
\hline 51. & Z-10-Tetradecen-1-ol acetate & NF & 17.373 & $\mathrm{C} 16 \mathrm{H} 30 \mathrm{O} 2$ & 10 \\
\hline 52. & Dodecanamide, N,N-bis(2-hydroxyethyl) & NF & 17.737 & $\mathrm{C} 16 \mathrm{H} 33 \mathrm{NO} 3$ & 10 \\
\hline 53. & 5,8-Dimethyl-1,2,3,4-tetrahydro-1-naphthol & NF & 17.847 & $\mathrm{C} 12 \mathrm{H} 16 \mathrm{O}$ & 3 \\
\hline 54. & 3-Hexen-1-ol, benzoate, (Z) & NF & 17.917 & $\mathrm{C} 13 \mathrm{H} 16 \mathrm{O} 2$ & 10 \\
\hline 55. & Nonanoic acid & Pelargic acid & 18.014 & $\mathrm{C} 9 \mathrm{H} 18 \mathrm{O} 2$ & 10 \\
\hline 56. & Nonanedioic acid, monomethyl ester & NF & 18.153 & $\mathrm{C} 10 \mathrm{H} 18 \mathrm{O} 4$ & 10 \\
\hline 57. & (-)-Caryophyllene oxide & Caryophyllene oxide & 18.311 & $\mathrm{C} 15 \mathrm{H} 24 \mathrm{O}$ & 10 \\
\hline 58. & (+-)-Andirolactone & Andirolactone & 18.513 & $\mathrm{C} 11 \mathrm{H} 14 \mathrm{O} 2$ & 10 \\
\hline 59. & Ledol & NF & 18.64 & $\mathrm{C} 15 \mathrm{H} 26 \mathrm{O}$ & 10 \\
\hline 60. & (. + -.)-2-Methyl-6-p-tolyl-4-heptanol (diastereoisomer II) & NF & 18.693 & $\mathrm{C} 15 \mathrm{H} 24 \mathrm{O}$ & 9 \\
\hline 61. & Propanal, 2,2-dimethyl & NF & 18.777 & $\mathrm{C} 5 \mathrm{H} 10 \mathrm{O}$ & 1 \\
\hline 62. & 2,6,10-Trimethylundecan-(5E)-2,5,9-trien-4-one & NF & 18.869 & $\mathrm{C} 14 \mathrm{H} 22 \mathrm{O}$ & 10 \\
\hline 63. & 7-oxabicyclo[4.1.0]heptane, 1-(1,3-dimethyl-1,3-butadienyl)-2,2,6-trimethyl- & NF & 19.004 & $\mathrm{C} 15 \mathrm{H} 24 \mathrm{O}$ & 10 \\
\hline 64. & Octanoic acid, 6,6-dimethoxy-, methyl ester & NF & 19.087 & $\mathrm{C} 11 \mathrm{H} 22 \mathrm{O} 4$ & 10 \\
\hline 65. & 2-(p-methylphenyl)-2-nitropropane & NF & 19.212 & $\mathrm{C} 10 \mathrm{H} 13 \mathrm{NO} 2$ & 10 \\
\hline 66. & Azelaic Acid & Anchoic acid & 19.589 & $\mathrm{C} 9 \mathrm{H} 16 \mathrm{O} 4$ & 4 \\
\hline 67. & cis-9-oxabicyclo[6.1.0]non-2-ene & NF & 19.736 & $\mathrm{C} 8 \mathrm{H} 12 \mathrm{O}$ & 10 \\
\hline 68. & 1-Buten-3-one, 1-(2-carboxy-4,4-dimethylcyclobutenyl) & NF & 19.864 & $\mathrm{C} 11 \mathrm{H} 14 \mathrm{O} 3$ & 10 \\
\hline 69. & Campherenone & Campherenone & 20.056 & $\mathrm{C} 15 \mathrm{H} 24 \mathrm{O}$ & 10 \\
\hline 70. & 11-Hexadecyn-1-ol & NF & 20.231 & $\mathrm{C} 16 \mathrm{H} 300$ & 10 \\
\hline 71. & Cyclodecene, 1-ethyl-2-methyl- & NF & 20.385 & $\mathrm{C} 13 \mathrm{H} 24$ & 10 \\
\hline 72. & 1,3-Dioxolane-4,5-dicarboxylic acid, 2,2-dimethyl-, dimethyl ester & NF & 20.627 & $\mathrm{C} 9 \mathrm{H} 14 \mathrm{O} 6$ & 5 \\
\hline 73. & 10-(1-Methylallyl)tricyclo[6.3.1.0(2,7)]dodeca-2(7),3,5-trien-10-ol & NF & 20.768 & $\mathrm{C} 16 \mathrm{H} 200$ & 4 \\
\hline 74. & 2-Acetoxy-1,1,10-trimethyl-6,9-epidioxydecalin & NF & 20.894 & $\mathrm{C} 15 \mathrm{H} 24 \mathrm{O} 4$ & 10 \\
\hline 75. & Farnesyl Acetone C & Farnesyl Acetone & 21.18 & $\mathrm{C} 18 \mathrm{H} 300$ & 10 \\
\hline 76. & 17-Octadecynoic acid & NF & 21.401 & $\mathrm{C} 18 \mathrm{H} 32 \mathrm{O} 2$ & 10 \\
\hline 77. & Tetradecanoic acid & Myristic acid & 21.82 & $\mathrm{C} 14 \mathrm{H} 28 \mathrm{O} 2$ & 10 \\
\hline 78. & Driminol & Drimenol & 22.167 & $\mathrm{C} 15 \mathrm{H} 26 \mathrm{O}$ & 10 \\
\hline 79. & 2,2,6-Trimethyl-1-(3-methylbuta-1,3-dienyl)-7-oxabicyclo[4.1.0] heptan-3-ol & NF & 22.272 & $\mathrm{C} 14 \mathrm{H} 22 \mathrm{O} 2$ & 10 \\
\hline 80. & 1,3,5-trimethyl-6-methyliden-tricyclo[3.2.1.0(2,7)]oct-3-en-8-endo-ol & NF & 22.677 & $\mathrm{C} 12 \mathrm{H} 16 \mathrm{O}$ & 9 \\
\hline 81. & 1-Methyl-2-acetyl-6-methoxy-3,4-dihydronaphthalene & NF & 22.933 & $\mathrm{C} 14 \mathrm{H} 16 \mathrm{O} 2$ & 10 \\
\hline 82. & N-(1-Cyanoethyl)(7,7-dimethyl-2-oxobicyclo[2.2.1] hept-1-ylmethanesulfonamide & NF & 23.386 & $\mathrm{C} 13 \mathrm{H} 2 \mathrm{~N} 2 \mathrm{O} 3 \mathrm{~S}$ & 10 \\
\hline 83. & 5-(ethylamino)-1,6-dimethyl-2(1H)-quinolinone & NF & 23.511 & $\mathrm{C} 13 \mathrm{H} 16 \mathrm{~N} 2 \mathrm{O}$ & 10 \\
\hline 84. & $(-)-$ Isolongifolol & Isolongifolol & 23.926 & $\mathrm{C} 15 \mathrm{H} 26 \mathrm{O}$ & 10 \\
\hline 85. & Neophytadiene & Neophytadiene & 24.02 & $\mathrm{C} 2 \mathrm{OH} 38$ & 10 \\
\hline 86. & Naphthalene, 1-(1,1-dimethylethyl)-7-methoxy- & NF & 24.123 & $\mathrm{C} 15 \mathrm{H} 18 \mathrm{O}$ & 2 \\
\hline 87. & 2-Pentadecanone, 6,10,14-trimethyl & NF & 24.218 & $\mathrm{C} 18 \mathrm{H} 36 \mathrm{O}$ & 10 \\
\hline 88. & 2,5,8-Trimethyltricyclo[5.3.1.1 (3,9)]dodecane-2-anti,8-tnti-diol & NF & 24.561 & $\mathrm{C} 15 \mathrm{H} 26 \mathrm{O} 2$ & 3 \\
\hline 89. & Pentadecanoic acid & Pentadecyclic acid & 24.74 & $\mathrm{C} 15 \mathrm{H} 30 \mathrm{O} 2$ & 0 \\
\hline
\end{tabular}


Table 3 List of components of essential oil of Rumexhastatus (Continued)

\begin{tabular}{|c|c|c|c|c|c|}
\hline 90. & 9,19-Cycloergost-24(28)-en-3-ol, 4,14-dimethyl-, acetate & NF & 25.047 & $\mathrm{C} 32 \mathrm{H} 52 \mathrm{O} 2$ & 4 \\
\hline 91. & 8-Keto-10-dehydrobrominated-.beta.-snyderol & NF & 25.298 & $\mathrm{C} 15 \mathrm{H} 22 \mathrm{O} 2$ & 2 \\
\hline 92. & Widdrol & Widdrol & 25.848 & $\mathrm{C} 15 \mathrm{H} 26 \mathrm{O}$ & 10 \\
\hline 93. & 2,4,7,9-Tetramethyl-5-decyne-4,7-diol & NF & 26.036 & $\mathrm{C} 14 \mathrm{H} 26 \mathrm{O} 2$ & 4 \\
\hline 94. & Phenol, 2-methyl-4-(1,1,3,3-tetramethylbutyl) & NF & 26.54 & $\mathrm{C} 15 \mathrm{H} 24 \mathrm{O}$ & 10 \\
\hline 95. & Benzene, 1,1'-(1,2-diethyl-1,2-ethanediyl)bis[4-methoxy- & NF & 26.548 & $\mathrm{C} 2 \mathrm{OH} 26 \mathrm{O} 2$ & 10 \\
\hline 96. & (1R,3S)-2,2,3-Trimethyl-6-methylidenecyclohexane-1-carbaldehyde & NF & 26.624 & $\mathrm{C} 11 \mathrm{H} 18 \mathrm{O}$ & 5 \\
\hline 97. & Hexadecanoic acid, methyl ester & Methyl palmitate & 26.732 & $\mathrm{C} 17 \mathrm{H} 34 \mathrm{O} 2$ & 10 \\
\hline 98. & 1-Hexadecen-3-ol, 3,5,11,15-tetramethyl- & NF & 27.371 & $\mathrm{C} 2 \mathrm{OH} 40 \mathrm{O}$ & 10 \\
\hline 99. & Benzo[e]isobenzofuran-1,4-dione, 1,3,4,5,5a,6,7,8,9,9a-decahydro-6,6,9a-trime.. & NF & 27.585 & $\mathrm{C} 15 \mathrm{H} 20 \mathrm{O} 3$ & 10 \\
\hline 100. & Hexadecanoic acid & Palmitic acid & 27.984 & $\mathrm{C} 16 \mathrm{H} 32 \mathrm{O} 2$ & 10 \\
\hline 101. & Butane-1,1-dicarbonitrile, 1-cyclohexyl-3-methyl- & NF & 28.431 & $\mathrm{C} 13 \mathrm{H} 2 \mathrm{ON} 2$ & 10 \\
\hline 102. & 2-Methyl-2-propyl-2,5-dihydrofuran & NF & 28.552 & $\mathrm{C} 8 \mathrm{H} 14 \mathrm{O}$ & 10 \\
\hline 103. & 5A-Methyl-3,8-dimethylene-2-oxododecahydrooxireno[2',3':6,7]naphtho[1,2-b]fu... & NF & 28.643 & $\mathrm{C} 2 \mathrm{OH} 24 \mathrm{O} 5$ & 10 \\
\hline 104. & 4-(3,7,7-Trimethyl-2-oxabicyclo[3.2.0]hept-3-en-1-yl)but-3-en-2-one & NF & 28.98 & $\mathrm{C} 13 \mathrm{H} 18 \mathrm{O} 2$ & 10 \\
\hline 105. & Cyclobutanecarboxylic acid, 2-methyloct-5-yn-4-yl ester & NF & 29.064 & $\mathrm{C} 14 \mathrm{H} 22 \mathrm{O} 2$ & 10 \\
\hline 106. & Cyclooctenone, dimer & NF & 29.439 & $\mathrm{C} 16 \mathrm{H} 24 \mathrm{O} 2$ & 10 \\
\hline 107. & Undecane, 6-cyclohexyl- & NF & 29.639 & $\mathrm{C} 17 \mathrm{H} 34$ & 10 \\
\hline 108. & 2,4,5,7-Tetramethyl-2,6-octadiene & NF & 30.471 & $\mathrm{C} 12 \mathrm{H} 22$ & 10 \\
\hline 109. & Cyclohexane, 1,2,3,4,5,6-hexaethyl & NF & 30.77 & $\mathrm{C} 18 \mathrm{H} 36$ & 10 \\
\hline 110. & Cyclopentanone, 3-methyl-2-(2-pentenyl)- & NF & 31.291 & $\mathrm{C} 11 \mathrm{H} 18 \mathrm{O}$ & 10 \\
\hline 111. & 2-Propanon & Acetone & 31.44 & $\mathrm{C} 3 \mathrm{H} 6 \mathrm{O}$ & 10 \\
\hline 112. & beta.-Ionol \$\$3-Buten-2-ol, 4-(2,6,6-trimethyl-1-cyclohexen-1-yl)- & NF & 31.703 & $\mathrm{C} 13 \mathrm{H} 22 \mathrm{O}$ & 10 \\
\hline 113. & Velleral & Velleral & 32.121 & $\mathrm{C} 15 \mathrm{H} 20 \mathrm{O} 2$ & 10 \\
\hline 114. & 2-Hydrazino-2-imidazoline & NF & 32.733 & $\mathrm{C} 3 \mathrm{H} 8 \mathrm{~N} 4$ & 10 \\
\hline 115. & 2H-cyclopropa[g]benzofuran, 4,5,5A,6,6A,6B-hexahydro-4,4,6b-trimethyl-2-(1-m... & NF & 33.658 & $\mathrm{C} 15 \mathrm{H} 22 \mathrm{O}$ & 10 \\
\hline 116. & Hexadecane & Cetane & 37.132 & $\mathrm{C} 16 \mathrm{H} 34$ & 10 \\
\hline 117. & Docosane & Docosane & 38.808 & $\mathrm{C} 22 \mathrm{H} 46$ & 10 \\
\hline 118. & 4,4-6-Trimethyl-7-oxabicyclo[4.1.0]heptan-2-one & NF & 39.247 & $\mathrm{C} 9 \mathrm{H} 14 \mathrm{O} 2$ & 10 \\
\hline 119. & 1,2-Benzenedicarboxylic acid, bis(2-ethylhexyl) ester & NF & 39.623 & $\mathrm{C} 24 \mathrm{H} 38 \mathrm{O} 4$ & 10 \\
\hline 120. & 4-Allyl-1-ethoxy-3-phenylbenzo[c]-(1,2)-oxaphosphinine - 1-Oxide & NF & 40.4 & $\mathrm{C} 19 \mathrm{H} 1903 \mathrm{P}$ & 3 \\
\hline 121. & Hexadecane & Cetane & 41.915 & $\mathrm{C} 16 \mathrm{H} 34$ & 10 \\
\hline 122. & Undecane, 3,8-dimethyl- & NF & 44.76 & $\mathrm{C} 13 \mathrm{H} 28$ & 10 \\
\hline 123. & 4-Methyl-7-ethylizidine \$\$ 8-Methyl-5-ethylindolizidine & NF & 58.237 & $\mathrm{C} 11 \mathrm{H} 21 \mathrm{~N}$ & 10 \\
\hline
\end{tabular}

$0.1 \mathrm{~mL}$ of each concentration was added to the $3 \mathrm{~mL}$ of DPPH solution. The mixture obtained was incubated at $23{ }^{\circ} \mathrm{C}$ for $30 \mathrm{~min}$ in dark. After incubation the absorbance of each sample were recorded at the wavelength of $517 \mathrm{~nm}$ using double beam spectrophotometer. Ascorbic acid was used as positive control. All the samples were processed in triplicates and the percent activity was recorded as mean \pm SEM. The percent radical scavenging potential was figured out using the following formula;

$$
\begin{aligned}
\% \text { scavenging } & =\frac{\text { absorption of control-absorption of test sample }}{\text { absorption of control }} \\
& \times 100 \quad
\end{aligned}
$$

\section{ABTS radical scavenging assay}

The 2, 2-azinobis [3-ethylbenzthiazoline]-6-sulfonic acid (ABTS) free radicals scavenging assay of the essential oil was evaluated followed standard procedure [11]. ABTS solution $7 \mathrm{mM}$ and potassium persulfate solution $2.45 \mathrm{mM}$ were prepared and mixed thoroughly. The 


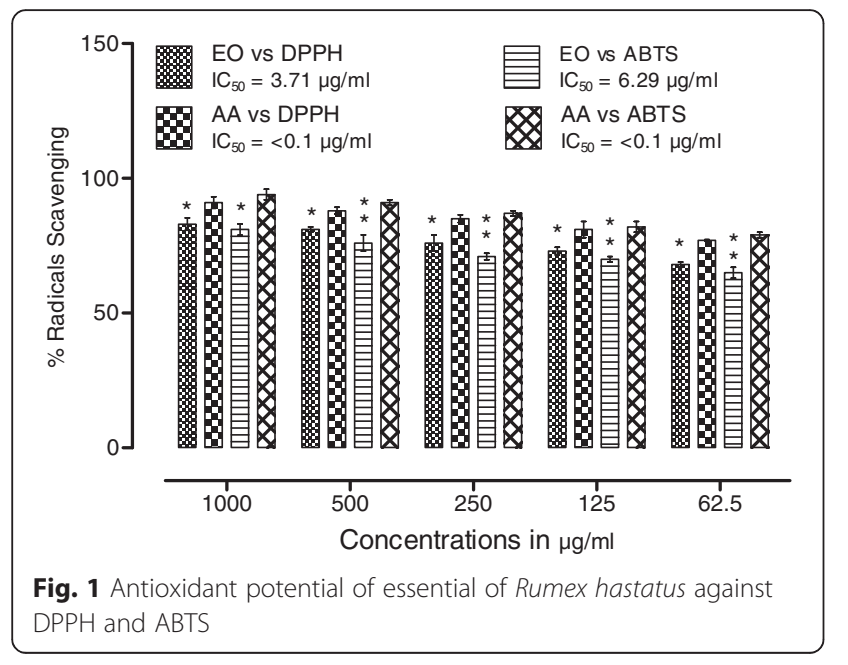

solution prepared was put in dark overnight for the production of free radicals. After incubation time the absorbance of solution was adjusted at $745 \mathrm{~nm}$ to 0.7 by the addition of $50 \%$ methanol. Test samples having volume of $300 \mu \mathrm{l}$ was taken in a test tube and $3 \mathrm{~mL}$ ABTS solution was added to it. The solution was transferred to the cuvette and absorbance values were taken for six minutes using double beam spectrophotometer. Ascorbic acid was used as positive control. All the samples were run in triplicate and percent ABTS radical scavenging potential was figured out using the following formula;

$$
\begin{aligned}
\% \text { scavenging activity } & =\frac{\text { control absorbance-sample absorbance }}{\text { control absorbance }} \\
& \times 100
\end{aligned}
$$

\section{Estimation of $\mathrm{IC}_{50}$ values}

The median inhibitory concentration i.e., $\mathrm{IC}_{50}$ values of AChE, BChE, DPPH and ABTS were determined by a linear regression analysis of the percent inhibition versus the concentrations of test samples through MS Excel program.

\section{Statistical data analysis}

All the tests were conducted in triplicate and the values were tabulated as mean \pm S.E.M. Significant difference of the percent inhibition of various test samples was analyzed via two way ANOVA following Bonferroni's post test using GraphPad Prism software in which the $P<0.05$ were considered significant.
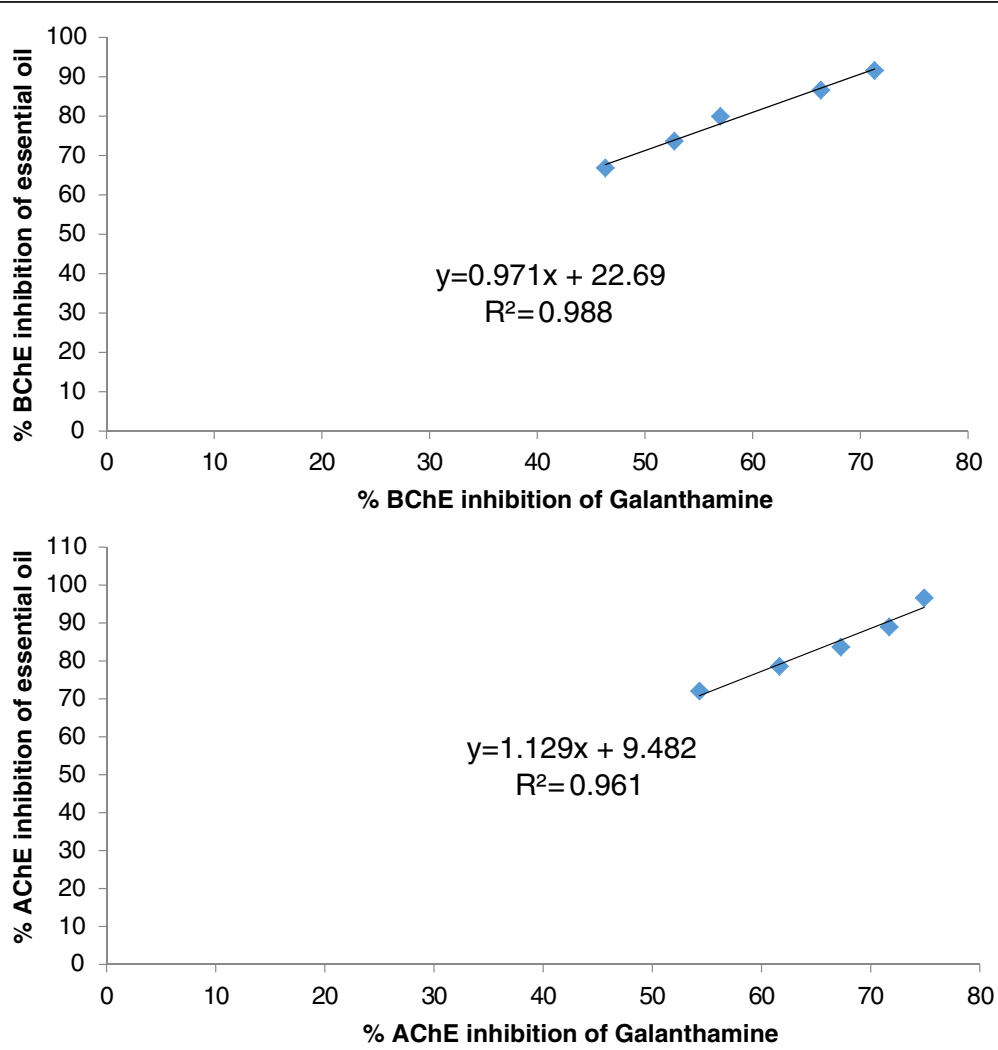

Fig. 2 a. Regression and correlation of percent BChE inhibition of essential oil Vs Galanthamine. b. Regression and correlation of percent AChE inhibition of essential oil Vs Galanthamine 


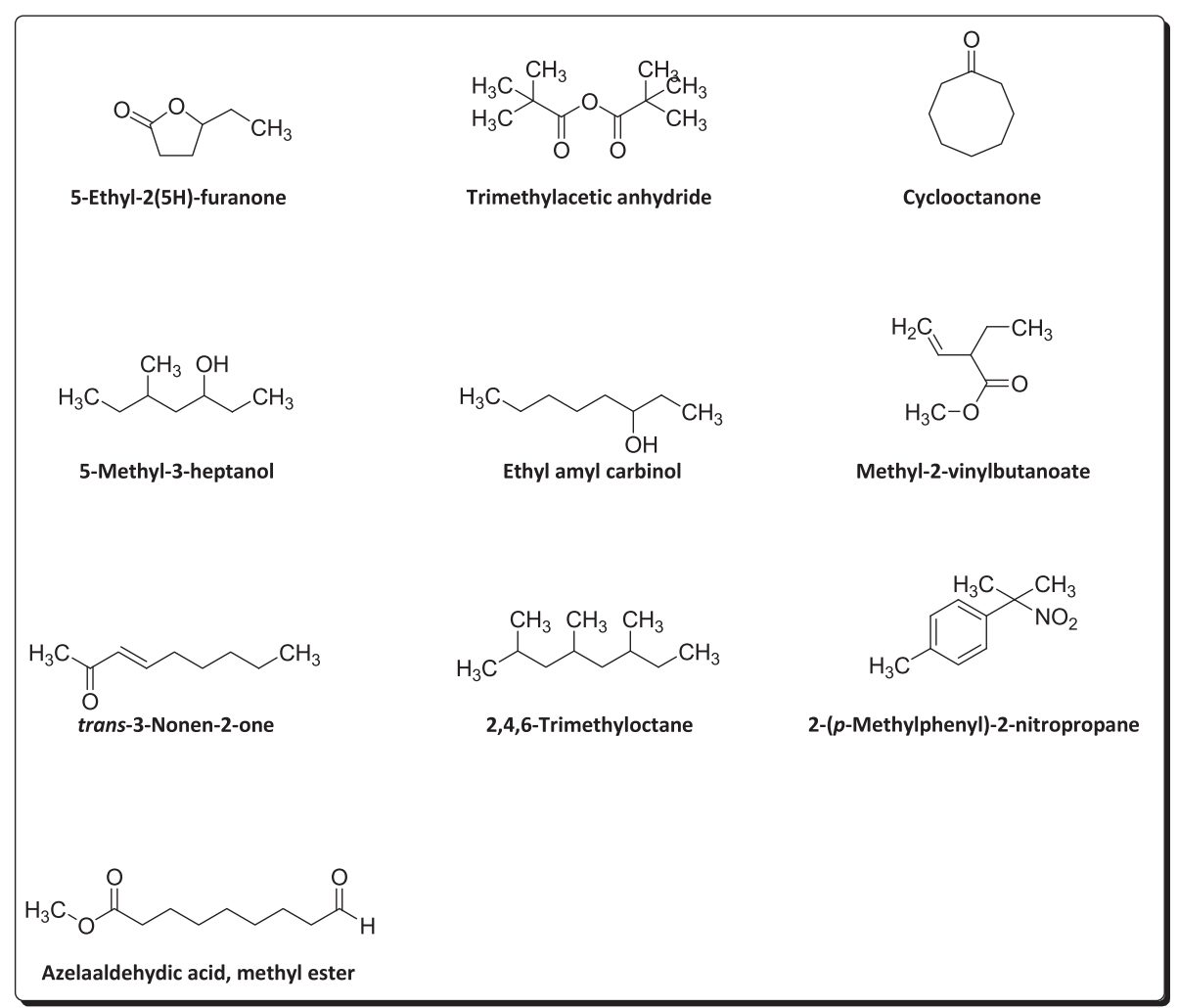

Fig. 3 Structures of some important components of essential oil of Rumex hastatus

\section{Results and discussion}

In the current investigational study the radical scavenging potential of volatile oil was studied based on spectrophotometric analysis. The sources of free radicals employed were DPPH and ABTS, which have maximum absorbance values at $517 \mathrm{~nm}$ and $745 \mathrm{~nm}$ respectively. After getting scavenged by antioxidant compounds the colors of DPPH (violet) and ABTS (blue) solution change into yellow. Change in the color results in decrease of absorbance values which is directly proportional to the amount of radical scavenging compounds in the solution [32, 33].

Similarly, the anticholinesterase activity is based on the hydrolysis of acetylthiocholine iodide and butyrylcholine iodide by the formation of the yellow 5-thio-2nitrobenzoate anion as a result of the reaction of DTNB with thiocholines, catalyzed by enzymes at a wavelength of $412 \mathrm{~nm}$ using spectrophotometer or microplate reader. Acetylthiocholine iodide and butyrylthiocholine iodide work as substrate of the reaction, while the DTNB is utilized for the measurement of cholinesterase activity. The percent inhibition of enzymatic activity is calculated from the rate of change in absorption of the reaction mixture [34].

The available literature on etiology of diseases demonstrate multiple causative agents responsible for specific disease [35]. In the context of Alzheimer's disease, numerous investigators have reported the role of various causative agents along with various successful approaches [36]. Like all neurodegenerative disorders, the free radicals have a prominent role in the induction and progression of $\mathrm{AD}$ [37]. By avoiding or attenuating the causative agents one can hinder the progression of a specific disease. In case of neurodegenerative disorders, the scavenging of free radicals can be a vital target. Various researchers have demonstrated the effective role of natural antioxidants especially the essential oils to combat the free radicals [38]. Similarly, one of the most widely employed treatment strategies for $\mathrm{AD}$ i.e., the inhibition of AChE to increase the concentration of neurotransmitter is highly recommended [39]. In this regard, essential oils are being investigated by advanced researchers with better results. Essential oils obtained from various plants possess marked anti-Alzheimer's potential due to the presence of wide variety of valuable compounds in it $[40,41]$. The anticholinesterase potential of essential oil of Rumex hastatus has been summarized in the Table 1, while the Table 2 shows various parameters of the compounds present in the essential oil of this plant. The GC-MS analysis of essential oil of $R$. hastatus demonstrates a total of 123 components as shown in Table 3. The anticholinesterase activity of essential oil of 


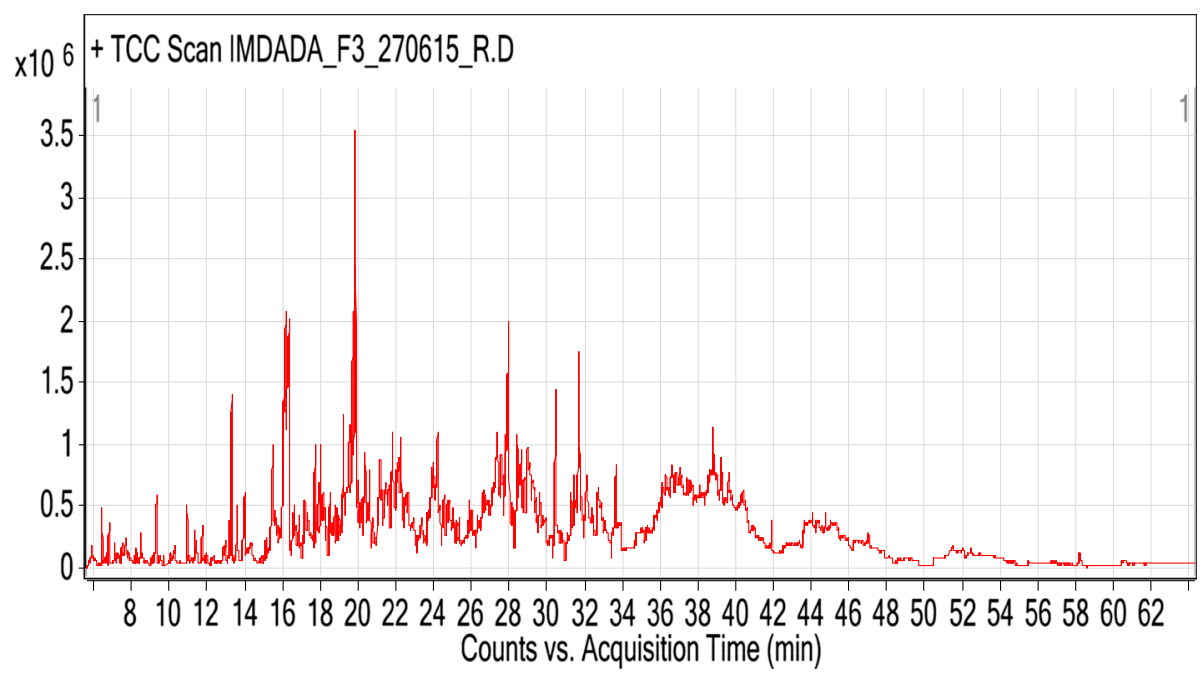

Fig. 4 GC-MS Chromatogram of essential oil of Rumex hastatus

$R$. hastatus might be due to its hydrophobic nature because of the good affinity of hydrophobic active site of AChE $[42,43]$. Some of the most common components of essential oils i.e., palmitic acid, myristic acid, pelargic acid, capric acid, docosane, cetane, velleral, acetone, methyl palmitate, widdrol, isolongifolol, ophytadiene, drimenol and levulinic acid have been found in the essential oil of $R$. hastatus. Some of these components have been reported previously by other investigators to possess antioxidant and anticholinesterase potentials [44-49]. The percent antioxidant potential of essential oil is illustrated in the Fig. 1. The peaks given in the Table 2 shows various volatile compounds like 5-ethyl$2(5 \mathrm{H})$-furanone, trimethylacetic anhydride, cyclooctanone, 5-methyl-3-heptanol, methyl 2-vinylbutanoate, 2( $p$-methylphenyl)-2-nitropropne, azelaaldehydic acid, 2,4,6-trimethyloctane and trans-3-nonen-2-one with retention times of $6.447,6.818,10.958,11.363,11.761$, $12.97,13.171,13.308,15.063$ and 19.213 min respectively. Going to the detail of various components of essential oil of $R$. hastatus, it is clear that the marked anticholinesterase potential shown by essential oil is observed due to the presence of wide variety of compounds in it. Essential oil demonstrated 74.90, 71.70, 67.26, 61.64, 54.32 \% AChE inhibition at 1000, 500, 250, 125, $62.5 \mu \mathrm{g} / \mathrm{ml}$ respectively. Similarly, the BChE inhibition exhibited by essential oil was recorded as 71.32, 66.33, $46.32,52.73,57.00 \%$ at $1000,500,250,125,62.5 \mu \mathrm{g} / \mathrm{ml}$ respectively. The essential oil attain $\mathrm{IC}_{50}$ values of 32.54 and $97.38 \mu \mathrm{g} / \mathrm{ml}$ for $\mathrm{AChE}$ and $\mathrm{BChE}$ inhibitions respectively. The anticholinesterase potential shown by essential oil goes parallel with the positive control which is also obvious from the Fig. 2 ( $\mathrm{a} \& \mathrm{~b}$ ) with the correlation coefficient of 0.961 and 0.988 for essential oil versus $\mathrm{AChE}$ and $\mathrm{BChE}$ respectively. Apart from the anticholinesterase potential of essential oil, the antioxidant potential of essential oil of various plants has been reported with discrimination by various investigators $[50,51]$. In our current investigational study, the free radicals scavenging assay of essential oil of $R$. hastatus against DPPH and ABTS was significant and almost comparable with the positive control. From Fig. 1, it is clear that essential oil exhibited marked potential with $\mathrm{IC}_{50}$ of 3.71 and $6.29 \mu \mathrm{g} / \mathrm{ml}$ against DPPH and ABTS respectively, which is also comparable with the previously reported literature. The previously reported data of $R$. hastatus verifies its anticholinesterase and antioxidant potentials which may be linked to the current investigational studies [26]. Some important components of essential oil and the chromatogram have been given in Figs. 3 and 4 respectively.

\section{Conclusion}

Essential oil isolated for the first time from the $R$. hastatus and its chemical composition demonstrates that $R$. hastatus is a source of valuable volatile components. Based on the anticholinesterase and antioxidant results of essential oil, it can be concluded that $R$. hastatus plant may be an effective source of compounds which may lead to possible palliative therapy and cure of oxidative stresses and neurodegenerative diseases.

\section{Competing interests}

All authors declare that they have no competing interests.

\section{Authors' contributions}

FU, AS and MRS conceived the idea and did literature survey. SA, MA, IA and AZ conducted practical work. MI and FU analyzed the data. All the authors contributed in drafting of the manuscript. 


\section{Acknowledgements}

All authors are thankful to Dr. Ali Hazrat, Department of Botany, Shaheed Benazir Bhutto University Sheringal, Dir (U), Pakistan for the identification of plant.

\section{Author details}

'Department of Pharmacy, University of Malakand, Chakdara 18000, Dir (L), KPK, Pakistan. ${ }^{2}$ International Center for Chemical and Biological Sciences, H.E.J. Research Institute of Chemistry University of Karachi, Karachi, Pakistan. ${ }^{3}$ Department of Pharmacy, Kohat University of Science \&Technology, Kohat, Pakistan.

Received: 1 October 2015 Accepted: 12 January 2016 Published: 25 January 2016

\section{References}

1. Van Wyk B-E, Wink M. Medicinal plants of the world: an illustrated scientific guide to important medicinal plants and their uses: Timber Press; Portland, USA 2004.

2. Samant S, Palni L, Kumar S, Kukreja A, Dwivedi S, Singh A. Diversity, distribution and indigenous uses of essential oil-yielding medicinal plants of the Indian Himalayan region. J Med Arom Plant Sci. 2000;22(1B):671-84.

3. Ruberto G, Baratta MT. Antioxidant activity of selected essential oil components in two lipid model systems. Food Chem. 2000;69(2):167-74.

4. Kumar H, Lim H-W, More SV, Kim B-W, Koppula S, Kim IS, et al. The role of free radicals in the aging brain and Parkinson's disease: convergence and parallelism. Int J Mol Sci. 2012;13(8):10478-504.

5. Mukherjee PK, Kumar V, Mal M, Houghton PJ. In vitro acetylcholinesterase inhibitory activity of the essential oil from Acorus calamus and its main constituents. Planta Med. 2007;73(3):283.

6. Small GW, Rabins PV, Barry PP, Buckholtz NS, DeKosky ST, Ferris SH, et al. Diagnosis and treatment of Alzheimer disease and related disorders: consensus statement of the American Association for Geriatric Psychiatry, the Alzheimer's Association, and the American Geriatrics Society. JAMA. 1997;278(16):1363-71.

7. Jensen SJK. Oxidative stress and free radicals. J Mol Struct. 2003;666:387-92.

8. Sadiq A, Mahmood F, Ullah F, Ayaz M, Ahmad S, Haq FU, et al. Synthesis, anticholinesterase and antioxidant potentials of ketoesters derivatives of succinimides: a possible role in the management of Alzheimer's. Chem Cent J. 2015;9(1):31

9. Masella R, Di Benedetto R, Vari R, Filesi C, Giovannini C. Novel mechanisms of natural antioxidant compounds in biological systems: involvement of glutathione and glutathione-related enzymes. J Nutr Biochem. 2005;16(10): 577-86.

10. Ayaz M, Junaid M, Subhan F, Ullah F, Sadiq A, Ahmad S, et al. Heavy metals analysis, phytochemical, phytotoxic and anthelmintic investigations of crude methanolic extract, subsequent fractions and crude saponins from Polygonum hydropiper L. BMC Complement Altern Med. 2014;14(1):465.

11. Ullah F, Ayaz M, Sadiq A, Hussain A, Ahmad S, Imran M, et al. Phenolic, flavonoid contents, anticholinesterase and antioxidant evaluation of Iris germanica var; florentina. Nat Prod Res. 2015;13:1-5.

12. Ullah I, Subhan F, Ayaz M, Shah R, Ali G, Haq IU, et al. Anti-emetic mechanisms of Zingiber officinale against cisplatin induced emesis in the pigeon; behavioral and neurochemical correlates. BMC Complement Altern Med. 2015;15(1):34.

13. Sadiq A, Khan S, Shah SMH. Larvicidal, insecticidal, brine shrimp cytotoxicity and anti-oxidant activities of Diospyros kaki (L.) reported from Pakistan. Pak J Pharm Sci. 2015;28(4):1239-43.

14. Shah S, Sadiq A, Gul F. Antibacterial potential of methanolic extracts and sub-fractions of Teucrium stocksianum Bioss collected from Malakand division, Pakistan. Pharmacologyonline. 2015;1:8-12.

15. Shah S, Shah SMM, Ahmad Z, Yaseen M, Shah R, Sadiq A, et al. Phytochemicals, in vitro antioxidant, total phenolic contents and phytotoxic activity of Cornus macrophylla Wall bark collected from the North-West of Pakistan. Pak J Pharm Sci. 2015;28(1):23-8.

16. Shah SM, Sadiq A, Ullah F. Antioxidant, total phenolic contents and antinociceptive potential of Teucrium stocksianum methanolic extract in different animal models. BMC Complement Altern Med. 2014;14(1):181.

17. Zeb A, Sadiq A, Ullah F, Ahmad S, Ayaz M. Phytochemical and toxicological investigations of crude methanolic extracts, subsequent fractions and crude saponins of Isodon rugosus. Biol Res. 2014;47(1):57.
18. Zeb A, Sadiq A, Ullah F, Ahmad S, Ayaz M. Investigations of anticholinestrase and antioxidant potentials of methanolic extract, subsequent fractions, crude saponins and flavonoids isolated from Isodon rugosus. Biol Res. 2014;47(1):1-10.

19. Burits M, Bucar F. Antioxidant activity of Nigella sativa essential oil. Phytother Res. 2000;14(5):323-8.

20. Islam S, Nasrin S, Khan MA, Hossain AS, Islam F, Khandokhar P, et al. Evaluation of antioxidant and anticancer properties of the seed extracts of Syzygium fruticosum Roxb. growing in Rajshahi, Bangladesh. BMC Complement Altern Med. 2013;13(1):142.

21. Uddin MN, Afrin R, Uddin MJ, Alam AH, Rahman AA, Sadik G. Vanda roxburghii chloroform extract as a potential source of polyphenols with antioxidant and cholinesterase inhibitory activities: identification of a strong phenolic antioxidant. BMC Complement Altern Med. 2015;15(1):195.

22. Perry N, Perry E. Aromatherapy in the management of psychiatric disorders. CNS drugs. 2006;20(4):257-80.

23. Tita I, Mogosanu GD, Tita MG. Ethnobotanical inventory of medicinal plants from the South-West of Romania. Farmacia. 2009;57(2):141-56.

24. Uysal I, Onar S, Karabacak E, Çelik S. Ethnobotanical aspects of Kapıdağ Peninsula (Turkey). Biol Divers Conserv. 2010;3(3):15-22.

25. Ummara U, Bokhari TZ, Altaf A, Younis U, Dasti AA. Pharmacological Study of Shogran Valley Flora, Pakistan. Int J Sci Eng Res. 2013;4(9):1-9.

26. Ahmad S, Ullah F, Ayaz M, Sadiq A, Imran M. Antioxidant and anticholinesterase investigations of Rumex hastatus D. Don: potential effectiveness in oxidative stress and neurological disorders. Biol Res. 2015; 48(1):20.

27. Lambert R, Skandamis PN, Coote PJ, Nychas GJ. A study of the minimum inhibitory concentration and mode of action of oregano essential oil, thymol and carvacrol. J Appl Microbiol. 2001;91(3):453-62.

28. Stein S, Mirokhin D, Tchekhovskoi D, G M. The NIST Mass Spectral Search Program for the NIST/EPA/NIH Mass Spectra Library; Standard Reference Data Program of the National Institute of Standards and Technology: Gaithersburg, MD, USA. 2002.

29. Adams R. Identification of essential oil components by gas chromatography/mass spectrometry. Carol Stream: Allured Publishing; 2007. p. 804.

30. Ayaz M, Junaid M, Ahmed J, Ullah F, Sadiq A, Ahmad S, et al. Phenolic contents, antioxidant and anticholinesterase potentials of crude extract, subsequent fractions and crude saponins from Polygonum hydropiper $\mathrm{L}$. BMC Complement Altern Med. 2014;14(1):145.

31. Kamal Z, Ullah F, Ayaz M, Sadiq A, Ahmad S, Zeb A, et al. Anticholinesterse and antioxidant investigations of crude extracts, subsequent fractions, saponins and flavonoids of Atriplex laciniata L.: potential effectiveness in Alzheimer's and other neurological disorders. Biol Res. 2015;48(1):21.

32. Erel O. A novel automated direct measurement method for total antioxidant capacity using a new generation, more stable ABTS radical cation. Clin Biochem. 2004:37(4):277-85.

33. Bondet $\mathrm{V}$, Brand-Williams W, Berset $\mathrm{C}$. Kinetics and mechanisms of antioxidant activity using the DPPH. free radical method. LWT-Food Sci Technol. 1997;30(6):609-15.

34. Ellman GL, Courtney KD, Andres V, Featherstone RM. A new and rapid colorimetric determination of acetylcholinesterase activity. Biochem Pharmacol. 1961:7(2):88-95.

35. Chamberlin WM, Naser SA. Integrating theories of the etiology of Crohn's Disease On the etiology ofCrohn's Disease: Questioning the Hypotheses. Med Sci Monitor. 2006;12(2):RA27-33.

36. Cummings $\mathrm{J}$, Vinters HV, Cole GM, Khachaturian ZS. Alzheimer's disease Etiologies, pathophysiology, cognitive reserve, and treatment opportunities. Neurology. 1998;51(1 Suppl 1):S2-S17.

37. Valko M, Leibfritz D, Moncol J, Cronin MT, Mazur M, Telser J. Free radicals and antioxidants in normal physiological functions and human disease. Int J Biochem Cell Biol. 2007:39(1):44-84.

38. Devasagayam T, Tilak J, Boloor K, Sane KS, Ghaskadbi SS, Lele R. Free radicals and antioxidants in human health: current status and future prospects. Japi. 2004;52(794804):4

39. Trinh N-H, Hoblyn J, Mohanty S, Yaffe K. Efficacy of cholinesterase inhibitors in the treatment of neuropsychiatric symptoms and functional impairment in Alzheimer disease: a meta-analysis. JAMA. 2003;289(2):210-6.

40. Savelev S, Okello E, Perry N, Wilkins R, Perry E. Synergistic and antagonistic interactions of anticholinesterase terpenoids in Salvia lavandulaefolia essential oil. Pharmacol Biochem Behav. 2003;75(3):661-8. 
41. Albano SM, Lima AS, Miguel MG, Pedro LG, Barroso JG, Figueiredo AC. Antioxidant, anti-5-lipoxygenase and antiacetylcholinesterase activities of essential oils and decoction waters of some aromatic plants. Rec Nat Prod. 2012;6(1):35-48.

42. Steinberg GM, Mednick ML, Maddox J, Rice R, Cramer J. Hydrophobic binding site in acetylcholinesterase. J Med Chem. 1975;18(11):1056-61.

43. Loizzo MR, Tundis R, Menichini F, Menichini F. Natural products and their derivatives as cholinesterase inhibitors in the treatment of neurodegenerative disorders: an update. Curr Med Chem. 2008;15(12):1209-28.

44. Stamatis $H$, Sereti V, Kolisis F. Studies on the enzymatic synthesis of lipophilic derivatives of natural antioxidants. J Am Oil Chem Soc. 1999; 76(12):1505-10.

45. Mehendale S, Kilari A, Dangat K, Taralekar V, Mahadik S, Joshi S. Fatty acids, antioxidants, and oxidative stress in pre-eclampsia. Int J Gynecol Obstet. 2008;100(3):234-8

46. Decker EA, Warner K, Richards MP, Shahidi F. Measuring antioxidant effectiveness in food. J Agric Food Chem. 2005:53(10):4303-10.

47. Sengupta A, Ghosh M. Comparison of native and capric acid-enriched mustard oil effects on oxidative stress and antioxidant protection in rats. $\mathrm{Br}$ J Nutr. 2012;107(06):845-9.

48. Öztürk M, Duru ME, Kivrak Ş, Mercan-Doğan N, Türkoglu A, Özler MA. In vitro antioxidant, anticholinesterase and antimicrobial activity studies on three Agaricus species with fatty acid compositions and iron contents: A comparative study on the three most edible mushrooms. Food Chem Toxicol. 2011;49(6):1353-60.

49. Yi B-H, Kim D-H. Antioxidant activity of maltol, kojic acid, levulinic acid furfural, 5-hydroxymethyl furfural, and pyrazine. Korean J Food Sci Technol. 1982;14(3):265-70.

50. Tepe B, Daferera D, Sokmen A, Sokmen M, Polissiou M. Antimicrobial and antioxidant activities of the essential oil and various extracts of Salvia tomentosa Miller (Lamiaceae). Food Chem. 2005;90(3):333-40.

51. Ayaz M, Junaid M, Ullah F, Sadiq A, Khan MA, Ahmad W, et al. Comparative chemical profiling, cholinesterase inhibitions and anti-radicals properties of essential oils from Polygonum hydropiper L: A Preliminary anti-Alzheimer's study. Lipids Health Dis. 2015;14(1):141.

\section{Submit your next manuscript to BioMed Central and we will help you at every step:}

- We accept pre-submission inquiries

- Our selector tool helps you to find the most relevant journal

- We provide round the clock customer support

- Convenient online submission

- Thorough peer review

- Inclusion in PubMed and all major indexing services

- Maximum visibility for your research

Submit your manuscript at www.biomedcentral.com/submit 